\title{
PEPSI deep spectra
}

\section{Chemical analysis of the ancient planet-host star Kepler-444 ${ }^{\star}$}

\author{
C. E. Mack III ${ }^{1}$, K. G. Strassmeier ${ }^{1}$, I. Ilyin ${ }^{1}$, S. C. Schuler ${ }^{2}$, F. Spada ${ }^{1}$, and S. A. Barnes ${ }^{1}$ \\ ${ }^{1}$ Leibniz-Institute for Astrophysics Potsdam (AIP), An der Sternwarte 16, 14482 Potsdam, Germany \\ e-mail: kstrassmeier@aip.de \\ ${ }^{2}$ University of Tampa, Tampa, FL 33606, USA \\ e-mail: sschuler@ut.edu
}

Received 24 July 2017 / Accepted 15 December 2017

\begin{abstract}
Context. With the Large Binocular Telescope (LBT), we obtained a spectrum with PEPSI, its new optical high-resolution échelle spectrograph. The spectrum has very high resolution and a high signal-to-noise $(\mathrm{S} / \mathrm{N})$ and is of the K0V host Kepler-444, which is known to host five sub-Earth-sized rocky planets. The spectrum has a resolution of $R \approx 250000$, a continuous wavelength coverage from $4230 \AA$ to $9120 \AA$, and an S/N between 150-550:1 (blue to red).

Aims. We performed a detailed chemical analysis to determine the photospheric abundances of 18 chemical elements. These were used to place constraints on the bulk composition of the five rocky planets.

Methods. Our spectral analysis employs the equivalent-width method for most of our spectral lines, but we used spectral synthesis to fit a small number of lines that required special care. In both cases, we derived our abundances using the MOOG spectral analysis package and Kurucz model atmospheres.

Results. We find no correlation between elemental abundance and condensation temperature among the refractory elements $\left(T_{\mathrm{C}}>950 \mathrm{~K}\right)$. In addition, using our spectroscopic stellar parameters and isochrone fitting, we find an age of $10 \pm 1.5 \mathrm{Gyr}$, which is consistent with the asteroseismic age of $11 \pm 1 \mathrm{Gyr}$. Finally, from the photospheric abundances of $\mathrm{Mg}, \mathrm{Si}$, and $\mathrm{Fe}$, we estimate that the typical Fe-core mass fraction for the rocky planets in the Kepler-444 system is approximately $24 \%$.

Conclusions. If our estimate of the Fe-core mass fraction is confirmed by more detailed modeling of the disk chemistry and simulations of planet formation and evolution in the Kepler-444 system, then this would suggest that rocky planets in more metal-poor and $\alpha$-enhanced systems may tend to be less dense than their counterparts of comparable size in more metal-rich systems.
\end{abstract}

Key words. stars: abundances - stars: atmospheres - stars: late-type - stars: activity - planetary systems

\section{Introduction}

The chemical composition of a planet-hosting star is known to influence the architecture of its planetary system (Dawson \& Murray-Clay 2013; Adibekyan et al. 2013; Beaugé \& Nesvorný 2013). Statistical surveys of exoplanet host stars show that Jupiter-like giants are preferentially formed around metal-rich stars (Fischer \& Valenti 2005; Udry \& Santos 2007; Ghezzi et al. 2010), while smaller planets appear to be less selective (Sousa et al. 2011; Buchhave et al. 2012, 2014; Schuler et al. 2015). This has been interpreted as evidence for the prevalence of the core accretion model of planet formation (Ida \& Lin 2004; Mordasini et al. 2009). However, it should be noted that recent work (Nayakshin 2017) has shown that formation by gravitational instability via the tidal downsizing

\footnotetext{
${ }^{\star}$ Based on data acquired with PEPSI using the Large Binocular Telescope (LBT). The LBT is an international collaboration among institutions in the United States, Italy, and Germany. LBT Corporation partners are the University of Arizona on behalf of the Arizona university system; Istituto Nazionale di Astrofisica, Italy; LBT Beteiligungsgesellschaft, Germany, representing the Max-Planck Society, the Leibniz-Institute for Astrophysics Potsdam (AIP), and Heidelberg University; the Ohio State University; and the Research Corporation, on behalf of the University of Notre Dame, University of Minnesota and University of Virginia.
}

model can also reproduce the observed correlation between giant planets and host star metallicity.

On the other hand, the formation of terrestrial planets does not seem to prefer iron-rich environments and succeeds with similar frequency around stars of various metallicities. Nevertheless, recent studies have hinted at some chemical preference for terrestrial planets. In particular, Adibekyan et al. (2015) showed that metal-poor planet hosts tend to have a higher $[\mathrm{Mg} / \mathrm{Si}]$ ratio than metal-poor stars without detected planets. In addition, Dressing et al. (2015) found that for a small sample of Kepler planets with well-known masses and radii, planets with masses $<6 M_{\oplus}$ are likely to have very similar iron-to-magnesium silicate ratios, and therefore follow a single mass-radius relationship. Furthermore, Santos et al. (2015) used a simplified chemical network based on detailed simulations performed by other teams (Lodders 2003; Seager et al. 2007) to estimate the Fe-core mass fraction of a subset of the rocky planets from the Dressing et al. sample: Kepler-10b, Kepler-93b, and CoRoT-7b. Santos et al. (2015) found that their simplified model predicted Fe-core mass fractions of $\sim 30 \%$ for all three planets. In this paper, we aim to use the same simplified model to estimate the Fe-core mass fraction for the five rocky planets orbiting the Kepler host star Kepler-444.

The discovery of a multi-planet system around Kepler-444 (KOI-3158, HIP 94931) was reported in Campante et al. (2015). 
Their photometric analysis revealed five transiting planets with radii between those of Mercury and Venus and orbits within $0.1 \mathrm{AU}$ of the star (i.e., within the orbit of Mercury). Perhaps even more astounding is the old age of the host star, 11.2 $\pm 1.0 \mathrm{Gyr}$, which Campante et al. determined from asteroseismology. Furthermore, their spectroscopic analysis of a Keck/HIRES spectrum $(R \approx 60000, S / N \approx 200)$ yielded $T_{\text {eff }}=5046 \mathrm{~K}$ and $\log g=4.6$, together with sub-solar abundances of $\mathrm{Fe}$ as well as $\mathrm{Si}$ and $\mathrm{Ti}$ (two $\alpha$-elements), leading to a moderately high $[\alpha / \mathrm{Fe}]$ index of $0.26 \mathrm{dex}$. Thus, it appears that (low-mass) planet formation has been ongoing shortly after the Universe was created and that the chemical composition of the prestellar material did not have to be metal rich.

In order to perform a detailed chemical abundance analysis of Kepler-444, we obtained high-resolution, high signal-tonoise $(\mathrm{S} / \mathrm{N})$ spectra with the optical high-resolution échelle spectrograph PEPSI (Strassmeier et al. 2015), which was recently commissioned at the $11.8 \mathrm{~m}$ Large Binocular Telescope (Hill et al. 2012). We performed our detailed analysis in order to ascertain the degree to which the photospheric abundances of the host star can place constraints on the bulk compositions of the planets.

In Sect. 2 we describe the observations and the spectrograph, while Sect. 3 describes the data analysis. Section 4 presents our results, and in Sect. 5 we discuss the implications of these results and suggest possible research avenues for future work. Finally, in Sect. 6, we summarize our findings and conclusions.

\section{Observations}

High-resolution $R \approx 250000$ spectra of Kepler-444 were obtained with the Potsdam Echelle Polarimetric and Spectroscopic Instrument (PEPSI; Strassmeier et al. 2015) at the $2 \times 8.4 \mathrm{~m} \mathrm{LBT}$. PEPSI is an asymmetric fiber-fed white-pupil échelle spectrograph with two arms (blue and red) that typically cover the wavelength range $383-907 \mathrm{~nm}$. The instrument is stabilized in a pressure- and thermally controlled chamber and is fed by three pairs of octagonal fibres per telescope. The different core diameters of the fibres and their respective imageslicer width set the three different resolutions of the spectrograph (43 000, 120000 , and 250 000). The 250 000-mode was used for the spectra in this paper and is made possible with a seven-slice image slicer and a $100 \mu \mathrm{m}$ fibre through a projected sky aperture of 0.74 ", comparable to the median seeing of the LBT site. The actual resolution varies with wavelength and depends on the position of the échelle order on the CCD and on the focus position. It is generally lowest at the order edges, in particular in the blue arm, and highest near the blaze in the red arm at $7000 \AA$. It ranges from an average of 220000 in the blue arm to an average of 250000 in the red arm. The projected image slices are covered in the cross-dispersion direction by 12 pixels per slice. Two $10.3 \mathrm{k} \times 10.3 \mathrm{k}$ STA1600LN CCDs with $9 \mu \mathrm{m}$ pixels record a total of 92 échelle orders.

PEPSI can be used either with sky fibers for simultaneous sky and target exposures or with light from a stabilized FabryPérot etalon (FPE) for simultaneous fringe and target exposures for precise radial velocities. All spectra in this paper were taken in target+target mode, that is, no simultaneous sky or FPE exposures, only two simultaneous target spectra, one from each of the LBT unit telescopes. At the time when the present spectra were obtained, we had just repositioned the cross dispersers and ended up with an extra échelle order in the redmost unit. Because of (telescope) time constraints, we were unable to use the bluest cross disperser at all. Therefore our Kepler-444 spectra cover the wavelength range 4230-9120 $\mathrm{A}$, with an average dispersion that varies from $7 \mathrm{~m} \AA$ per pixel in CD I to $19 \mathrm{~m} \AA$ per pixel in CD VI.

The data we study here were taken during spectrograph commissioning, and thus full instrument performance was not achieved yet (see paper I and paper II; Strassmeier et al. 2018a,b for details). Observations were conducted on May 23, and 24, 2015. Exposure times of 7, 10, 20, and $30 \mathrm{~min}$ were chosen, depending upon wavelength range, seeing, and time availability. Table 1 summarizes the observing log. Basic data reduction followed the procedures laid out and described in detail in paper I.

After optimal extraction and wavelength calibration, the onedimensional spectra were coadded to build the single "deep spectrum" in Table 1. Altogether, the deep spectrum was assembled from seven individual spectra in CD II, five in CD III, four in CD IV, three in CD V, and four in CD VI. Each pixel of each spectrum was weighted according to its inverse variance from the optimal extraction and was optimally resampled before adding. We also note that each individual spectrum is two spectra in our case because the LBT is in fact a combination of two telescopes. At one occasion on May 24, 09:46UT, the DX M1 mirror cell panicked during integration, and the spectrum from one side was lost. Figure 1a shows the full spectrum. A portion of it spanning the wavelength region $\lambda 6140-\lambda 6170$ is shown in Fig. $1 \mathrm{~b}$.

\section{Analysis}

The chemical abundances (relative to solar) of 18 elements were derived from the deep spectrum of Kepler-444. The 2014 version of the local thermal equilibrium (LTE) spectral analysis package MOOG (Sneden 1973) was used to perform the spectral analysis. The abundances were derived from measurements of the equivalent widths (EWs) of atomic lines using the SPECTRE analysis package (Fitzpatrick \& Sneden 1987). By requiring excitation and ionization balance of the $\mathrm{Fe}$ I and $\mathrm{Fe}$ II lines, we were able to derive the stellar parameters: $T_{\mathrm{eff}}, \log g$, $[\mathrm{Fe} / \mathrm{H}]$, and $\xi$ (microturbulence). The line lists for the wavelength regions encompassing each element were taken from the Vienna Atomic Line Database VALD; Piskunov et al. (1995); Kupka et al. (1999). Abundances are always given relative to solar ${ }^{1}$. The solar spectrum that we used is the grand average from paper I (Strassmeier et al. 2018a).

The carbon abundances were derived from the $\mathrm{C}_{2}$ Swan system features at $\lambda 5086$ and $\lambda 5135$ using the synth driver in MOOG to synthetically fit them by eye. In addition, the EWs of three high-excitation $C_{I}$ lines were measured: $\lambda 5052.17$, 15380.34, and $\lambda 7113.18$. Abundances were derived from these EW measurements using the MOOG abfind driver.

The two components of the $\mathrm{C}_{2}$ Swan system yield relative abundances that are identical, that is, $[\mathrm{C} / \mathrm{H}]=-0.56$. However, the high-excitation $\mathrm{C}$ I lines yield discrepant relative abundances of $[\mathrm{C} / \mathrm{H}]=-0.31,-0.07,+0.22$, respectively. Previous LTE analyses of high-excitation lines, such as the O I triplet at $\lambda 7775 \AA$ (e.g., Schuler et al. 2004, 2006; Ramírez et al. 2014), have found that the abundances derived from high-excitation lines increase with decreasing $T_{\text {eff }}$ for stars with $T_{\text {eff }} \leq 5400 \mathrm{~K}$. For the highexcitation C I lines in particular, Schuler et al. (2015) found that these lines also give abundances that are higher by $\sim 0.16 \mathrm{dex}$ than the $\mathrm{C}_{2}$ Swan lines for Kepler-37, a star with $T_{\text {eff }}$ of $5400 \mathrm{~K}$.

$1[X / \mathrm{H}]=\log \left(N_{X}\right)_{\text {star }}-\log \left(N_{X}\right)_{\text {Sun }}$ with $\log \left(N_{X}\right)=\log \left(N_{X} / N_{\mathrm{H}}\right)+12$. 
Table 1. Spectra observed with PEPSI.

\begin{tabular}{|c|c|c|c|c|c|c|c|c|c|c|}
\hline $\begin{array}{l}\text { Date } \\
\text { (UT) }\end{array}$ & Time & Air mass & $\begin{array}{l}\text { Seeing } \\
\left({ }^{\prime \prime}\right)\end{array}$ & $\begin{array}{l}\text { Exp. } \\
\text { (min) }\end{array}$ & $\mathrm{CD}$ & $\begin{array}{l}\lambda \\
(\mathrm{nm})\end{array}$ & $\mathrm{S} / \mathrm{N}^{a}$ & $\mathrm{CD}$ & $\begin{array}{l}\lambda \\
(\mathrm{nm})\end{array}$ & $\mathrm{S} / \mathrm{N}^{a}$ \\
\hline \multirow[t]{6}{*}{ 2015, May 24} & 08:19 & 1.1 & 0.9 & 20 & III & $480-544$ & 85 & $\mathrm{~V}$ & $628-742$ & 156 \\
\hline & $08: 40$ & 1.1 & 0.9 & 20 & III & $480-544$ & 98 & V & $628-742$ & 181 \\
\hline & $09: 24$ & 1.1 & 0.9 & 20 & III & $480-544$ & 99 & V & $628-742$ & 202 \\
\hline & $09: 46$ & 1.1 & 0.9 & 20 & II & $423-480$ & 75 & $\ldots$ & $\ldots$ & \\
\hline & $10: 14$ & 1.1 & 0.9 & 20 & II & $423-480$ & 92 & VI & $742-912$ & 262 \\
\hline & $10: 35$ & 1.1 & 0.9 & 7 & II & $423-480$ & 54 & VI & $742-912$ & 156 \\
\hline \multirow[t]{6}{*}{ 2015, May 25} & $03: 51$ & 1.2 & 0.9 & 20 & II & $423-480$ & 98 & IV & $742-912$ & 177 \\
\hline & $07: 58$ & 1.2 & 0.8 & 30 & III & $480-544$ & 142 & VI & $742-912$ & 247 \\
\hline & $08: 29$ & 1.2 & 0.8 & 30 & III & $480-544$ & 164 & VI & $742-912$ & 284 \\
\hline & 09:02 & 1.1 & 1.0 & 10 & II & $423-480$ & 60 & IV & $544-628$ & 131 \\
\hline & $10: 32$ & 1.1 & 1.0 & 30 & II & $423-480$ & 133 & IV & $544-628$ & 261 \\
\hline & $11: 03$ & 1.1 & 1.0 & 30 & II & $423-480$ & 134 & IV & $544-628$ & 265 \\
\hline \multirow[t]{3}{*}{ Deep spectrum } & & & & & I & & $\ldots$ & IV & $544-628$ & $300-500$ \\
\hline & & & & & II & $423-480$ & $150-300$ & $\mathrm{~V}$ & $628-742$ & $250-300$ \\
\hline & & & & & III & $480-544$ & $200-300$ & VI & $742-912$ & $550-300$ \\
\hline
\end{tabular}

Notes. ${ }^{(a)}$ The $\mathrm{S} / \mathrm{N}$ for the individual spectra is given for the central wavelength of each cross disperser (CD). The S/N for the deep spectrum is given for the full wavelength range as indicated.

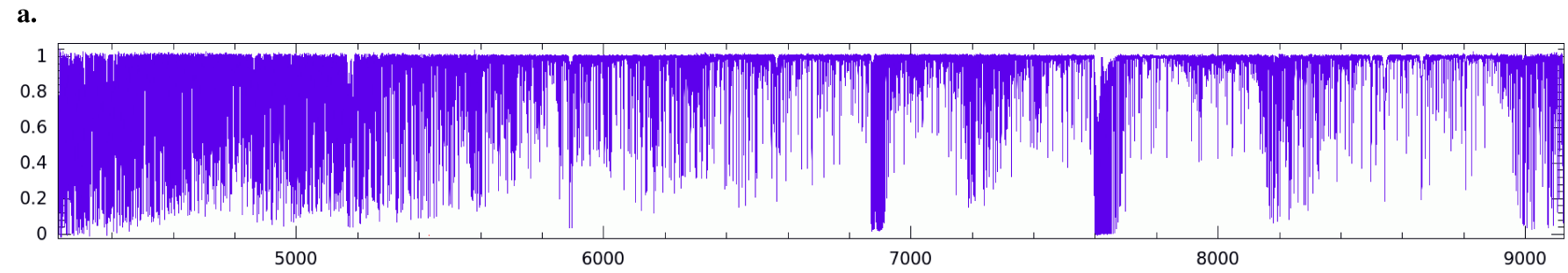

b.

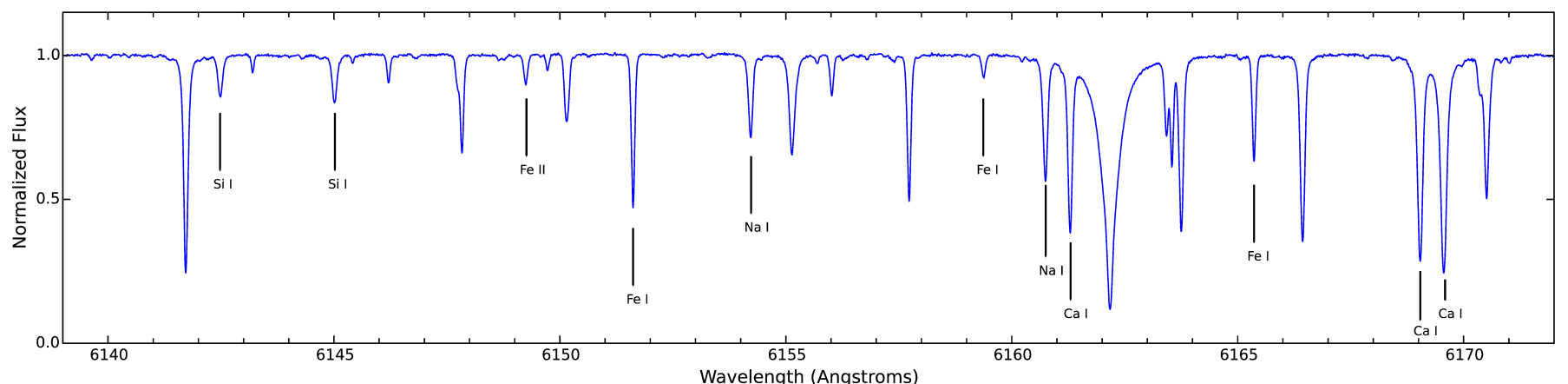

Fig. 1. Kepler-444 deep spectrum. a) Full spectral region between 4230-9120 A. $b$ ) Spectral region between $\sim 6140 \AA$ and $\sim 6170 \AA$ A A selection of the lines measured in this region is labeled.

Our C abundances for Kepler-444 seem to follow this same pattern, suggesting that in this cool star, the $\mathrm{C}_{\mathrm{I}}$ lines do not provide reliable abundances. We therefore adopt the abundance determined from the $\mathrm{C}_{2}$ Swan system as the carbon abundance here.

For the oxygen abundances, four spectral lines were analyzed: the forbidden line at $\lambda 6300.34$, and the near-infrared triplet at $\lambda 7771.94, \lambda 7774.17$, and $\lambda 7775.39$. The forbidden line was analyzed by measuring the equivalent width and using the MOOG blends driver to account for known blends with spectral lines from other elements, in particular, the known blend with an Ni line. For the near-infrared triplet, we measured their equivalent widths and used the standard MOOG abfind driver. We then corrected the near-infrared triplet abundances for NLTE effects using the corrections from Takeda (2003). The forbidden line and the near-infrared triplet yielded relative abundances that agree reasonably well: $[\mathrm{O} / \mathrm{H}]=-0.12$ and -0.09 , respectively. More recently, Amarsi et al. (2016) provided three-dimensional (3D) NLTE corrections for 1D LTE abundances for the temperature range 5000-6500 K. Applied to our measurements, this corrects to abundances of -0.13 and -0.11 for the forbidden and the near-infrared triplet, respectively. Therefore we simply 
Table 2. Stellar parameters and abundances.

\begin{tabular}{ll}
\hline \hline & Kepler-444 \\
\hline$T_{\text {eff }}(\mathrm{K})$ & \\
$\log g(\mathrm{cgs})$ & $5,172 \pm 75$ \\
$\xi\left(\mathrm{km} \mathrm{s}^{-1}\right)$ & $1.64 \pm 0.37$ \\
{$[\mathrm{C} / \mathrm{H}]$} & $-0.56 \pm 0.000^{a} \pm 0.03^{b}$ \\
{$[\mathrm{O} / \mathrm{H}]^{c}$} & $-0.11 \pm 0.027 \pm 0.12$ \\
{$[\mathrm{Na} / \mathrm{H}]$} & $-0.44 \pm 0.055 \pm 0.08$ \\
{$[\mathrm{Mg} / \mathrm{H}]$} & $-0.27 \pm 0.033 \pm 0.05$ \\
{$[\mathrm{Al} / \mathrm{H}]$} & $-0.19 \pm 0.014 \pm 0.05$ \\
{$[\mathrm{Si} / \mathrm{H}]$} & $-0.37 \pm 0.022 \pm 0.04$ \\
{$[\mathrm{~K} / \mathrm{H}]$} & $-0.38 \pm \ldots \pm 0.13$ \\
{$[\mathrm{Ca} / \mathrm{H}]$} & $-0.53 \pm 0.020 \pm 0.10$ \\
{$[\mathrm{Sc} / \mathrm{H}]$} & $-0.34 \pm 0.011 \pm 0.08$ \\
{$[\mathrm{Ti} / \mathrm{H}]$} & $-0.27 \pm 0.036 \pm 0.11$ \\
{$[\mathrm{~V} / \mathrm{H}]$} & $-0.18 \pm 0.024 \pm 0.11$ \\
{$[\mathrm{Cr} / \mathrm{H}]$} & $-0.46 \pm 0.006 \pm 0.07$ \\
{$[\mathrm{Fe} / \mathrm{H}]$} & $-0.52 \pm 0.011 \pm 0.12$ \\
{$[\mathrm{Mn} / \mathrm{H}]$} & $-0.61 \pm 0.030 \pm 0.10$ \\
{$[\mathrm{Co} / \mathrm{H}]$} & $-0.33 \pm 0.015 \pm 0.06$ \\
{$[\mathrm{Ni} / \mathrm{H}]$} & $-0.50 \pm 0.013 \pm 0.05$ \\
{$[\mathrm{Zn} / \mathrm{H}]$} & $-0.41 \pm 0.004 \pm 0.08$ \\
{$[\mathrm{Y} / \mathrm{H}]$} & $-0.51 \pm \ldots \pm 0.12$ \\
\hline
\end{tabular}

Notes. Adopted solar parameters (Cox 2000): $T_{\text {eff }}=5777 \quad \mathrm{~K}$, $\log g=4.44$, and $\xi=1.38 \mathrm{~km} \mathrm{~s}^{-1}$. ${ }^{(a)}$ The uncertainty in the mean. For $\mathrm{C}$, both spectral lines yielded the same abundance; for $\mathrm{K}$ and $\mathrm{Y}$, the abundance was derived from a single spectral line; and for Co, all the spectral lines yielded the same abundance, except for one that differed by 0.01 dex. ${ }^{(b)}$ The quadratic sum of the uncertainty in the mean and the uncertainties due to $T_{\text {eff }}, \log g$, and $\xi .{ }^{(c)}$ Corrected for NLTE effects, see text.

averaged these abundances and adopted an $[\mathrm{O} / \mathrm{H}]$ abundance of -0.11 . We note that significant disagreement between the nearinfrared triplet and forbidden line relative abundance has been seen before in open cluster dwarf stars with spectral types similar to Kepler-444 (Schuler et al. 2006). However, there is some evidence that the discrepancy between these two $[\mathrm{O} / \mathrm{H}]$ indicators may decrease with age, which we discuss further in Sect. 5.1.

For $\mathrm{V}$ and $\mathrm{Co}$, in order to account for possible hyper-fine structure (hfs) effects (Prochaska \& McWilliam 2000), we used the MOOG blends driver on specific spectral lines: the V I lines at $\lambda 6090.21$ and $\lambda 6111.65$, and the Co I lines at $\lambda 5301.04$, $\lambda 5352.04$, and $\lambda 6814.94$. The hfs components for most of these spectral lines were obtained from Johnson et al. (2006), but the components for $\lambda 5352.04$ came from Jofré et al. (2017). The solar-normalized abundances for these $\mathrm{V}$ and Co lines derived using the blends driver are in good agreement with the solarnormalized abundances derived from the $\mathrm{V}$ and $\mathrm{Co}$ lines that were analyzed with the standard MOOG abfind driver. The hfs effects for these lines therefore evidently have a negligible effect on the solar-normalized abundances, and the adopted $\mathrm{V}$ and Co abundances quoted in Table 2 are the mean of the abundances derived using the blends and abfind drivers. We also note that hfs affects mostly lines with larger EWs, which we found to be roughly $\geq 40 \mathrm{~m} \AA$.

For two elements, $\mathrm{K}$ and $\mathrm{Y}$, the abundance was derived from the measurements of a single spectral line. In order to obtain the best estimate of the total uncertainty in the abundance for these particular lines, we estimated how the uncertainty in the
EW-measurement affected the uncertainty in the abundance. For simplicity, a minimum-maximum method was used. For both lines, we chose three different continuum placements within the rms scatter of the local continuum, and for each continuum placement made four different measurements of the EW. This gave us a set of 12 EWs for each line in both the Kepler-444 and solar spectra. For each line, the maximum and mininum EW values were used to compute a maximum and minimum $\log \left(N_{X}\right)$. The differences between the maximum and minimum $\log \left(N_{X}\right)$ were adapted as the uncertainties in $\log \left(N_{X}\right)$ and propagated through the computation of the solar-normalized abundances. These uncertainties in the solar-normalized abundances (due to the uncertainty in the EWs) were then added in quadrature to the errors in the abundances due to the stellar parameters, in order to determine the final uncertainties quoted in Table 2. We also note that the sensitivity of the derived abundances to the adopted metallicity is small compared to the sensitivities to $T_{\text {eff }}, \log g$, and $\xi$.

We also determined the $\mathrm{Mn}$ abundance as another odd- $Z$ element. Mn is only weakly NLTE dependent, corrections are only 0.003 dex (Bergemann \& Gehren 2008), even less than for O, and thus we neglected NLTE effects for Mn. However, we have reliable hfs components (Johnson et al. 2006) for only three Mn lines $(\lambda 5432.546, \lambda 6013.513, \lambda 6021.819)$. We measured other Mn lines that we did not have hfs components for and found a large spread of up to -0.2 dex in the abundances, so we do not consider them to be reliable without hfs corrections. The average of the three lines is given in Table 2. In addition, we attempted to measure Li, but there was no spectral line distinguishable from the continuum noise at $\lambda 6707.8$. There were also no discernible spectral lines for the high-excitation $\mathrm{N}$ lines at $\lambda 7468.3, \lambda 8216.3$, and $\lambda 8683.4$.

The analysis we performed was nearly identical to the analysis of the planet-hosting wide binaries HD 20782/81 (Mack et al. 2014) and HD 80606/07 (Mack et al. 2016). The stellar parameters and relative abundances that we derived for Kepler-444 are summarized in Table 2. The adopted line list, EWs, and line-byline $\log N$ abundances of each element for Kepler-444 and the Sun are given in Table A.1.

\section{Results}

\subsection{Stellar parameters and abundances}

The stellar parameters $\left(T_{\text {eff }}=5172 \pm 75 \mathrm{~K}, \log g=4.56 \pm 0.18\right.$, $\xi=1.64 \pm 0.37 \mathrm{~km} \mathrm{~s}^{-1}$, and $\left.[\mathrm{Fe} / \mathrm{H}]=-0.52 \pm 0.12\right)$ and elemental abundances (Table 2) derived for Kepler-444 are consistent with it being a metal-poor $\sim \mathrm{K} 0$ dwarf. The $\log g$ value is identical to the one derived from asteroseismology by Campante et al. (2015), whereas our value for $T_{\text {eff }}$ differs from theirs by $126 \mathrm{~K}$. However, the two $T_{\text {eff }}$ values still agree within $3 \sigma$.

We also note here that given the $\approx 50$-day rotation period of Kepler-444 (see Sect. 4.3), a radius of $0.75 R_{\odot}$ translates into a maximum $v \sin i$ of $\approx 0.8 \mathrm{~km} \mathrm{~s}^{-1}$. This value is considerably lower than both our value for the microturbulence $\left(1.6 \mathrm{~km} \mathrm{~s}^{-1}\right)$ and the resolution of PEPSI $\left(\approx 1.3 \mathrm{~km} \mathrm{~s}^{-1}\right)$. We conclude that the spectral lines of Kepler-444 should not be significantly affected by rotational broadening.

Kepler-444 has significantly enhanced $\alpha$-abundances. To determine $[\alpha / \mathrm{Fe}]$ for Kepler-444, we averaged $[\mathrm{O} / \mathrm{H}],[\mathrm{Mg} / \mathrm{H}]$, $[\mathrm{Si} / \mathrm{H}],[\mathrm{Ca} / \mathrm{H}]$, and $[\mathrm{Ti} / \mathrm{H}]$ to obtain a value of $[\alpha / \mathrm{Fe}]=$ 0.23 . This value agrees well with the value of 0.26 that Campante et al. derived using their values for $[\mathrm{Si} / \mathrm{H}]$ and [Ti/H]. 


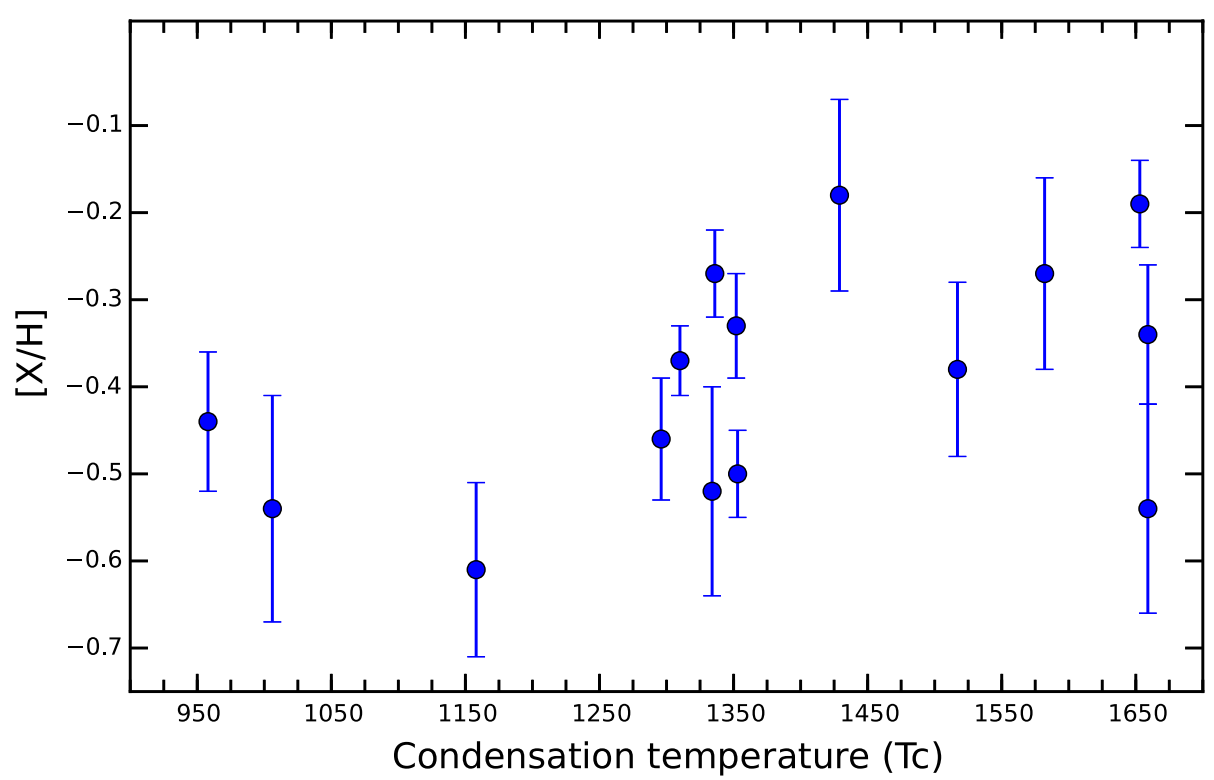

Fig. 2. Kepler-444 relative abundances vs. condensation temperature $\left(T_{\mathrm{C}}>950 \mathrm{~K}\right)$.
Previous studies (e.g., Meléndez et al. 2009; Ramírez et al. 2010; Schuler et al. 2011; Meléndez et al. 2014) have shown that Sun-like stars tend to show an increase in elemental abundance as a function of elemental condensation temperature, while the Sun itself appears to be depleted in elements with high condensation temperatures, that is, the refractory elements. Since it is these refractory elements that are the main constituents of rocky, terrestrial planets, it has been suggested (Meléndez et al. 2009; Ramírez et al. 2010) that the Sun being depleted in refractory elements may reflect the fact that these elements are locked up in the solar system's rocky planets. Therefore, given that Kepler-444 hosts five rocky planets, we investigated whether its abundances show any particular trend with condensation temperature.

Figure 2 shows the relative abundances of Kepler-444 vs. condensation temperature $\left(T_{\mathrm{C}}\right)$ for the refractory elements $\left(T_{\mathrm{C}}>950 \mathrm{~K}\right)$. These relative abundances are the means of the line-by-line differences between $\log N_{\text {Kepler-444 }}$ and $\log N_{\text {Sun }}$. For the condensation temperatures, we used the $50 \% T_{\mathrm{C}}$ values tabulated in Lodders (2003). There is no evident trend in the abundances as a function of condensation temperature. A simple linear regression fit returns an insignificant positive slope of $+0.00019 \pm 0.00012 \mathrm{dex} / \mathrm{K}$ with a standard fit deviation of 0.11 and chi-square of 0.21 . A Pearson correlation gives a coefficient of 0.393 , which for a sample size of 15 gives an adjusted correlation coefficient of only 0.07 .

In the following subsections, we describe several age indicators that we used to check for consistency with the asteroseismic age of $11 \pm 1$ Gyr. We also note that while each of the following age indicators have uncertainties greater than that of the asteroseismic age, each indicator does yield an age $(\sim 10-11 \mathrm{Gyr})$ that agrees well with the asteroseismic one, and that our main goal is to show that a variety of age indicators consistently produce an old age for Kepler-444.

\subsection{Yale-Potsdam Isochrones}

Given the spectroscopic $\log g$ and $T_{\text {eff }}$ we determined for Kepler-444, we used the Yale-Potsdam isochrones (YaPSI; Spada et al. 2017) to determine a mass and age for Kepler-444. We used the YaPSI models in conjunction with the Monte Carlo
Markov chain best-fit search tool BAGEMASS (Maxted et al. 2015). The $\chi^{2}$ minimization was performed with respect to the available observational constraints on the following parameters: $L / L_{\odot}, T_{\text {eff }}, \log g$, and $[\mathrm{m} / \mathrm{H}]$ (the alpha-enhanced rescaled metallicity; see below). Gravitational settling of He and heavy elements is also taken into account in the YaPSI models; this effect is important for a low-mass, old star such as Kepler-444. For Kepler-444, an initial $[\mathrm{m} / \mathrm{H}]$ of $\approx-0.30$ is indeed required to fit the present value of $[\mathrm{m} / \mathrm{H}]=-0.4$. Our best-fit solution yields a mass of $M=0.76 M_{\odot}$ and age of $10 \pm 1.5 \mathrm{Gyr}$. These values are in good agreement with those derived by Campante et al. (2015).

In order to take into account the enhancement in $\alpha$-elements $([\alpha / \mathrm{Fe}] \approx 0.2)$, we used the scaling relation of Salaris et al. (1993). Essentially, this scaling mimics the effects on the stellar models from the $\alpha$-enhanced abundances by introducing an effective rescaled metallicity $([\mathrm{m} / \mathrm{H}])$ that is slightly more metalrich than the spectroscopically determined metallicity $([\mathrm{Fe} / \mathrm{H}])$. These scaling relations are valid for stars that are sufficiently metal-poor (Kim et al. 2002). In the case of Kepler-444, with $[\alpha / \mathrm{Fe}] \approx 0.2$ and $[\mathrm{Fe} / \mathrm{H}]=-0.52$, we obtain a rescaled metallicity of $[\mathrm{m} / \mathrm{H}]=-0.40$; this is the value quoted in Fig. 3 . We note that the dashed lines in Fig. 3 represent YaPSI isochrones with the same $[\mathrm{m} / \mathrm{H}]$ for a range of ages that brackets the best-fit value of 10 Gyr. The shaded gray region is merely intended to guide the eye and highlight the locus defined by these isochrones; it is not a confidence interval.

\subsection{Gyrochronology}

Mazeh et al. (2015) have determined a rotation period of $49.4 \pm 6$ days for Kepler-444 from Kepler satellite data. Although the uncertainty in the period is large, it is typical for stars of such long periods in Kepler data, given the $\approx 90$ day length of each Kepler quarter. The relatively long period immediately indicates a fairly old star.

Stars of comparable mass to Kepler-444 have rotation periods of $\approx 24$ days in the 2.5 Gyr old open cluster NGC 6819 (Meibom et al. 2015), and $\approx 31$ days in the 4 Gyr old open cluster M 67 (Barnes et al. 2016). Extrapolation of the Skumanich-type rotation-age relationship to the period of Kepler-444 implies an age slightly in excess of $10 \mathrm{Gyr}$. 


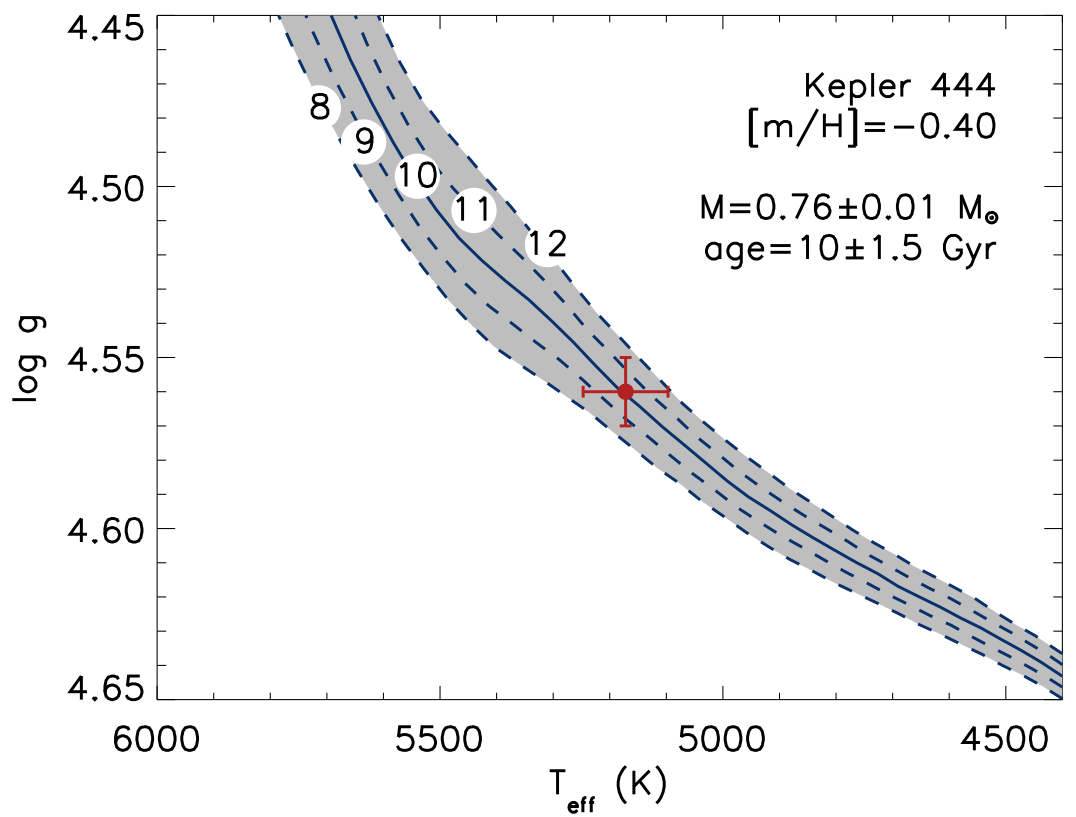

Fig. 3. Kepler-444 H-R diagram. The point with error bars corresponds to the $T_{\text {eff }}$ and $\log g$ of Kepler-444. The curves are the isochrones from YaPSI. The numbers in circles indicate the age in Gyr for each isochrone. The solid curve is the best-fit age.
A more precise formulation of gyrochronology, validated by reproducing the mass and age dependencies of rotation in a series of open clusters, including the 4 Gyr old open cluster M 67, is available in Barnes (2010). This formulation yields a gyro age for Kepler-444 of $10.9 \pm 2.5 \mathrm{Gyr}$, where we have used the convective turnover timescales tabulated in Barnes \& Kim (2010). The uncertainty in the gyro age is completely dominated by the relatively large uncertainty in the rotation period determination.

The gyro age is thus consistent with the asteroseismic determination, but has larger uncertainties in this particular case.

\section{4. $[\mathrm{Y} / \mathrm{Mg}]-$ clock}

We also measured $[\mathrm{Y} / \mathrm{H}]$ in order to take advantage of the [Y/Mg] age indicator for solar twins that was introduced by Nissen (2015) and further investigated by Tucci Maia et al. (2016). While Kepler-444 is certainly not a solar twin, we thought it would be interesting to see whether $[\mathrm{Y} / \mathrm{Mg}$ ] would yield an age consistent with the asteroseismic age and the other age indicators we employed. We determined that $[\mathrm{Y} / \mathrm{Mg}]=-0.19$ for Kepler-444, which, using the relation from Tucci Maia et al. (2016), corresponds to an age of $\approx 9$ Gyr. Given that their $[\mathrm{Y} / \mathrm{Mg}]$-age equation is specifically tuned for solar twins, this age estimate is not too discrepant with the $\approx 10-11 \mathrm{Gyr}$ ages determined from asteroseismology, gyrochronology, and the YaPSI isochrones. Recent studies (Feltzing et al. 2017; Delgado Mena et al. 2017) have shown that thick-disk stars with $[\mathrm{Fe} / \mathrm{H}]<-0.5$ do not show a tight correlation between age and [Y/Mg]. Therefore, as a member of the thick-disk population with an $[\mathrm{Fe} / \mathrm{H}]$ of -0.52 , Kepler-444 may be just beyond the limit of the range of applicability for the $[\mathrm{Y} / \mathrm{Mg}]$-clock.

\section{5. ${ }^{12} \mathrm{C} /{ }^{13} \mathrm{C}$ isotope ratio}

Finally, we derived an approximate value for the ${ }^{12} \mathrm{C} /{ }^{13} \mathrm{C}$ isotope ratio. As a zeroth-order approximation, we measured the equivalent widths of the $\mathrm{CN}$ features at $8003.5 \AA$ (due to ${ }^{12} \mathrm{C}$ ) and $8004.5 \AA$ (due to ${ }^{13} \mathrm{C}$ ). It was not possible to fit a simple Gaussian or Voigt profile to these features, so we integrated the area under the line profile using the Simpson rule integration procedure in MOOG. Repeated adjustments of where the wings of the spectral line merge with the continuum yielded the following equivalent widths for the two lines respectively; $5.2 \pm 0.1 \mathrm{~m} \AA$ and $3.0 \pm 0.4 \mathrm{~m} \AA$, where the error is the standard deviation in the measurements. Taking the ratio of these two values results in a very low isotope ratio of 1.7 , which is consistent with an old age of Kepler-444.

\section{Discussion}

\section{1. $C$ and $O$ abundances of Kepler-444}

The $\mathrm{C}$ and $\mathrm{O}$ abundances of Kepler-444 are consistent with the findings of Bensby \& Feltzing (2006) for stars with similar $[\mathrm{Fe} / \mathrm{H}]$. In their Fig. 11, it is clear that Kepler-444 lies near the edge of the distribution of stars with $[\mathrm{Fe} / \mathrm{H}] \approx-0.5$, but it is certainly within the overall scatter. However, in Delgado Mena et al. (2010) and Nissen et al. (2014), the C abundance of Kepler-444 falls below the general distribution of stars with $[\mathrm{Fe} / \mathrm{H}] \approx-0.5$. Still, given the scatter in the data for each of these studies, and the relatively few stars with metallicities near -0.5 (varying from 3-10 data points across the three studies), Kepler-444 cannot clearly be considered an outlier, but it has perhaps a relatively low $\mathrm{C}$ abundance compared to the stars with similar metallicities, kinematics, and $\alpha$-enhancement.

In addition, the lack of discrepancy in the relative oxygen abundances $([\mathrm{O} / \mathrm{H}])$ derived from the $[\mathrm{O} \mathrm{I}]-6300$ forbidden line and the near-infrared triplet is in agreement with the suggestion from Schuler et al. (2006) that this discrepancy disappears with age. In their studies of the oxygen abundances for the Pleiades, M 34, and Hyades open clusters, as well as for the UMa moving group (Schuler et al. 2004; King \& Schuler 2005, and Schuler et al. 2006), they found unexpectedly large O abundances derived from the near-infrared triplet among the $\mathrm{K}$ dwarfs in all three open clusters and in the UMa moving group. However, for the Hyades and the UMa moving group (which both have an age of about $600 \mathrm{Myr}$ ), the discrepancy was significantly smaller than in the Pleiades and M 34 (which have ages in the range 100-200 Myr). Therefore, the authors posited that the discrepancy may decrease with age, and the nearly identical O abundances derived from the $6300-\AA$ forbidden line and 
the near-infrared triplet in Kepler-444 may be the result of this trend. If this trend could be confirmed by studying the $\mathrm{O}$ abundances of $\mathrm{K}$ dwarfs in a statistical sample of open clusters and moving groups that span a wide range of ages, then this might become a powerful spectroscopic age indicator.

\subsection{How Kepler-444 compares to other known metal-poor planet hosts}

Adibekyan et al. (2012) showed that metal-poor host stars with small planets are enhanced in $\alpha$-elements with respect to metalpoor stars without known planets. Furthermore, Adibekyan et al. (2015) showed that small planets tend to prefer host stars with higher $[\mathrm{Mg} / \mathrm{Si}]$ ratios. Since Kepler-444 falls near the upper limits of the distributions shown in Fig. 4 of Adibekyan et al. (2015) and Fig. 2 of Adibekyan et al. (2012), the enhanced $\alpha$-abundance $([\alpha / \mathrm{Fe}]=0.23)$ and the comparatively high value of $[\mathrm{Mg} / \mathrm{Si}]=0.1$ for Kepler-444 are consistent with the findings from Adibekyan et al. (2012; 2015). Thus, Kepler-444 is a member of the growing population of metal-poor host stars that are enhanced in $\alpha$-elements and possess higher $[\mathrm{Mg} / \mathrm{Si}]$ than metal-poor non-hosts.

In addition, Adibekyan et al. (2015) suggested that since $[\mathrm{Mg} / \mathrm{Si}]$ has decreased over time as a result of Galactic chemical evolution, the interior structure and composition of older terrestrial planets, like those in the Kepler-444 system, might be significantly different from the terrestrial planets in the solar system. Compared to the solar system, the mass fraction of Fe may indeed have been considerably smaller, resulting in Earth-mass planets with significantly lower densities and correspondingly larger radii. In the next section, we estimate the possible Fecore mass fraction of the rocky planets in the Kepler-444 system using the photospheric chemical abundances.

\subsection{Estimating the iron core mass fraction from the photospheric abundances}

Here we follow the procedure and simplified chemical network used in Santos et al. (2015). This network consists of $\mathrm{H}, \mathrm{He}$, $\mathrm{C}, \mathrm{O}, \mathrm{Mg}, \mathrm{Si}$, and $\mathrm{Fe}$ because equilibrium condensation models (Lodders 2003; Seager et al. 2007) show that these seven elements are the most important for determining the major species of condensates in the protoplanetary disk, which are $\mathrm{H}_{2} \mathrm{O}, \mathrm{CH}_{4}$, $\mathrm{Fe}, \mathrm{MgSiO}_{3}$, and $\mathrm{Mg}_{2} \mathrm{SiO}_{4}$. Using the photospheric abundance ratios as proxies for the disk abundance ratios, we can relate our derived photospheric atomic abundances for $\mathrm{C}, \mathrm{O}, \mathrm{Mg}, \mathrm{Si}$, and $\mathrm{Fe}$ to the disk abundances of the five major condensates listed above. These disk abundances can be converted into mass fractions for each condensate. From these mass fractions, the Fe-core mass fraction of a rocky planet can be estimated by dividing the mass fraction of $\mathrm{Fe}$ by the combined mass fractions of $\mathrm{Fe}$ and the two magnesium silicates. From our calculations, we would typically expect an Fe-core mass fraction of approximately $24 \%$ for the rocky planets in the Kepler-444 system.

When interpreting this value for the Fe-core mass fraction, it is important to keep in mind the following caveats:

(a) This is only a first-order estimate using a simplified chemical network. A full numerical analysis using the photospheric abundances as inputs to determine which molecular and atomic species condense out of the disk should be performed in order to verify this result.

(b) Given that Kepler-444 is metal-poor and $\alpha$-enhanced, there may be other important disk condensates that are not included in the Santos et al. (2015) simplified chemical network, which is only valid for disk compositions where the $\mathrm{Mg}$ abundance is greater than the $\mathrm{Si}$ abundance.

(c) From the YaPSI isochrone analysis that we used to determine an age for Kepler-444, we found that due to gravitational settling, Kepler-444 may have initially been $\approx 0.1$ dex more metal-rich. The present-day photospheric abundances of Kepler-444 may therefore be less accurate proxies of the initial disk composition than is typically assumed for stars with solar-type composition.

Keeping these caveats in mind, this estimate of a typical Fe-core mass fraction of $\approx 24 \%$ for terrestrial planets in the Kepler-444 system, which is significantly less than the $\sim 30 \%$ Fe-core mass fraction observed in the solar system and predicted for other systems with solar-type composition (Santos et al. 2015), implies that rocky planets around metal-poor, $\alpha$-enhanced host stars may tend to be less dense than rocky planets of comparable size around more metal-rich host stars. A recent study (Santos et al. 2017) that estimated the composition of potential planets around a sample of 1111 stars observed with HARPS (Delgado Mena et al. 2017; Adibekyan et al. 2012), predicts that for old thick-disk stars, the Fe-core mass fraction for rocky planets would be 20-25\% (priv. comm. V. Adibekyan).

\section{Summary}

We have performed a detail chemical analysis of the planethosting star Kepler-444 using a high-resolution, high S/N spectrum obtained with the recently commissioned LBT/PEPSI spectrograph. We find that Kepler-444 is $\alpha$ enhanced and metal poor, in agreement with the initial spectroscopic analysis of Campante et al. (2015). Furthermore, using different age indicators, such as the YaPSI isochrones, gyrochronology, and the [Y/Mg] ratio, we find an age of around $10 \mathrm{Gyr}$ for Kepler-444, which is consistent with the $11 \pm 1$ Gyr that Campante et al. determined from their asteroseismic analysis.

In addition, we note that the good agreement between the relative (to solar) oxygen abundances determined from the [O I]-6300 forbidden line and the near-infrared O I-triplet in Kepler-444 may point toward a potentially powerful spectroscopic age indicator for $\mathrm{K}$ dwarfs, if the discrepancy usually observed between these two oxygen abundance indicators in young open clusters is seen to decrease with age in older open clusters.

Finally, we estimate the Fe-core mass fraction of a typical rocky planet in Kepler-444 system by using the photospheric abundances as proxies for the initial chemical composition of the disk. Our estimate of $\approx 24 \%$ suggests that rocky planets in metal-poor $\alpha$-enhanced systems may tend to be less dense than rocky planets of similar size in more metal-rich systems.

The reduced 1D deep spectra can be downloaded in FITS format from our web page at https://pepsi.aip.de. On demand, we also provide the data prior to continuum rectification.

Acknowledgements. We thank all engineers and technicians involved in PEPSI, in particular our late Emil Popow, who passed away much too early, as well as John Little and his LBTO mountain crew. Our thanks also go to Christian Veillet, LBT director, for enabling us to perform the observations. The many comments by our referee, V. Adibekyan, are also much appreciated. It led to an improved version of the paper. It is also our honor to thank the German Federal Ministry (BMBF) for the year-long support through their "boden-gebundene Verbundforschung" for LBT/PEPSI. 


\section{References}

Adibekyan, V. Z., Delgado Mena, E., Sousa, S. G., et al. 2012, A\&A, 547, A36 Adibekyan, V. Zh., Figueira, P., Santos, N. C., et al. 2013, A\&A, 560, A51 Adibekyan, V., Santos, N. C., Figueira, P., et al. 2015, A\&A, 581, L2

Amarsi, A. M., Asplund, M., Collet, R., \& Leenaarts, J. 2016, MNRAS, 455 3735

Barnes, S. A. 2010, ApJ, 722, 222

Barnes, S. A., \& Kim, Y.-C. 2010, ApJ, 721, 675

Barnes, S. A., Weingrill, J., Fritzewski, D., Strassmeier, K. G., \& Platais, I. 2016, ApJ, 823, 16

Beaugé, C., \& Nesvorný, D. 2013, ApJ, 763, 12

Bensby, T., \& Feltzing, S. 2006, MNRAS, 367, 1181

Bergemann, M., \& Gehren, T. 2008, A\&A, 492, 823

Buchhave, L. A., Latham, D. W., Johansen, A., et al. 2012, Nature, 486, 375

Buchhave, L. A., Bizzarro, M., Latham, D. W., et al. 2014, Nature, 509, 593

Campante, T. L., Barclay, T., Swift, J. J., et al. 2015, ApJ, 799, 170

Casagrande, L., Schönrich, R., Asplund, M., et al. 2011, A\&A, 530, A138

Cox, A. N. 2000, Allen's Astrophysical Quantities, 4th ed. (New York: AIP Press/Springer)

Dawson, R. I., \& Murray-Clay, R. A. 2013, ApJ, 767, L24

Delgado Mena, E., Israelian, G., González Hernández, J. I., et al. 2010, ApJ, 725, 2349

Delgado Mena, E., Tsantaki, M., Adibekyan, V. Zh., Sousa, S. G., Santos, N. C., González Hernández, J. I., \& Israelian, G. 2017, A\&A, 606, A94

Dressing, C. D., Charbonneau, D., Dumusque, X., et al. 2015, ApJ, 800, 135

Feltzing, S., Howes, L. M., McMillan, P. J., \& Stonkute, E. 2017, MNRAS, 465, L109

Fischer, D. A., \& Valenti, J. 2005, ApJ, 622, 1102

Fitzpatrick, M. J., \& Sneden, C. 1987, Bull. Am. Astron. Soc., 19, 1129

Ghezzi, L., Cunha, K., Smith, V. V., et al. 2010, ApJ, 720, 1290

Ida, S., \& Lin, D. N. C. 2004, ApJ, 616, 567

Hill, J., Green, R. F., Ashby, D. S., et al. 2012, SPIE, 8444, 1

Jofré, P., Heiter, U., Worley, C. C., et al. 2017, A\&A, 601, A38

Johnson, J. A., Ivans, I. I., \& Stetson, P. B. 2006, ApJ, 640, 801

Kim, Y.-C., Demarque, P., Yi, S. K., \& Alexander, D. R. 2002, ApJS, 143, 499

King, J. R., \& Schuler, S. C. 2005, PASP, 117, 911

Kupka, F., Piskunov, N., Ryabchikova, T. A., Stempels, H. C., \& Weiss, W. W. 1999, A\&AS, 138, 119

Lodders, K. 2003, ApJ, 591, 1220

Mack, C. E., III, Schuler, S. C., Stassun, K. G., \& Norris, J. 2014, ApJ, 787, 98
Mack, C. E., III, Stassun, K. G., Schuler, S. C., Hebb, L., \& Pepper, J. A. 2016, ApJ, 818, 54

Maxted, P. F. L., Serenelli, A. M., \& Southworth, J. 2015, A\&A, 575, A36

Mazeh, T., Perets, H. B., McQuillan, A., \& Goldstein, E. S. 2015, ApJ, 801, 3

Meibom, S., Barnes, S. A., Platais, I., et al. 2015, Nature, 517, 589

Meléndez, J., Asplund, M., Gustafsson, B., \& Yong, D. 2009, ApJ, 704, L66

Meléndez, J., Ramírez, I., Karakas, A. I., et al. 2014, ApJ, 791, 14

Mordasini, C., Alibert, Y., Benz, W., \& Naef, D. 2009, A\&A, 501, 1161

Nayakshin, S. 2017, PASA, 34, 2

Nissen, P. E. 2015, A\&A, 579, A52

Nissen, P. E., Chen, Y. Q., Carigi, L., Schuster, W. J., \& Zhao, G. 2014, A\&A, 568, A25

Piskunov, N. E., Kupka, F., Ryabchikova, T. A., Weiss, W. W., \& Jeffery, C. S 1995, A\&AS, 112, 525

Prochaska, J. X., \& McWilliam, A. 2000, ApJ, 537, L57

Ramírez, I., Asplund, M., Baumann, P., et al. 2010, A\&A, 521, A33

Ramírez, I., Meléndez, J., \& Asplund, M. 2014, A\&A, 561, A7

Salaris, M., Chieffi, A., \& Straniero, O. 1993, ApJ, 414, 580

Santos, N. C., Adibekyan, V., Mordasini, C., et al. 2015, A\&A, 580, L13

Santos, N. C., Adibekyan, V., Dorn, C., et al. 2017, A\&A, 608, A94

Seager, S., Kuchner, M., Hier-Majumder, C. A., \& Militzer, B. 2007, ApJ, 669, 1279

Schuler, S. C., King, J. R., Hobbs, L. M., \& Pinsonneault, M. H. 2004, ApJ, 602, L117

Schuler, S. C., King, J. R., Terndrup, D. M., et al. 2006, ApJ, 636, 432

Schuler, S. C., Flateau, D., Cunha, K., King, J. R., Ghezzi, L., \& Smith, V. V. 2011, ApJ, 732, 55

Schuler, S. C., Vaz, Z. A., Katime Santrich, O. J., et al. 2015, ApJ, 815, 5

Sneden, C. 1973, ApJ, 184, 839

Sousa, S. G., Santos, N. C., Israelian, G., Mayor, M., \& Udry, S. 2011, A\&A, 533, A141

Spada, F., Demarque, P., Kim, Y.-C., Boyajian, T. S., \& Brewer, J. M. 2017, ApJ, 838,161

Strassmeier, K. G., Ilyin, I., Jaervinen, A., et al. 2015, Astron. Nachr., 336, 324

Strassmeier, K. G., Ilyin, I., \& Steffen, M. 2018a, A\&A, 612, A44

Strassmeier, K. G., Ilyin, I., \& Weber, M. 2018b, A\&A, 612, A45

Takeda, Y. 2003, A\&A, 402, 343

Tucci Maia, M., Ramírez, I., Meléndez, J., et al. 2016, A\&A, 590, A32

Udry, S., \& Santos, N. C. 2007, ARA\&A, 45, 397

van Leeuwen, F. 2007, A\&A, 474, 653 
C. E. Mack III et al: PEPSI deep spectra. III.

Appendix A: Spectral lines we measured

Table A.1. Spectral lines we measured, equivalent widths (in mÅ), and abundances.

\begin{tabular}{|c|c|c|c|c|c|c|c|}
\hline Ion & $\begin{array}{c}\lambda \\
(\AA)\end{array}$ & $\begin{array}{c}\chi \\
(\mathrm{eV})\end{array}$ & $\begin{array}{c}\log g f \\
(-)\end{array}$ & $\begin{array}{l}\text { The Sun } \\
\mathrm{EW}_{\odot}\end{array}$ & $\log N_{\odot}$ & $\begin{array}{l}\text { Kepler-444 } \\
\text { EW }\end{array}$ & $\log N$ \\
\hline $\mathrm{C}$ I & 5052.17 & 7.69 & -1.304 & 37.2 & 8.53 & 10.0 & 8.22 \\
\hline $\mathrm{C}$ I & 5380.34 & 7.69 & -1.615 & 21.9 & 8.52 & 08.0 & 8.45 \\
\hline $\mathrm{C}_{\mathrm{I}}$ & 7113.18 & 8.65 & -0.685 & 22.9 & 8.49 & 12.1 & 8.71 \\
\hline [O I] & 6300.30 & 0.00 & -9.717 & 05.3 & 8.66 & 05.4 & 8.54 \\
\hline O I & 7771.94 & 9.15 & +0.369 & 69.3 & 8.72 & 25.2 & 8.59 \\
\hline O I & 7774.17 & 9.15 & +0.223 & 61.4 & 8.74 & 23.1 & 8.68 \\
\hline O I & 7775.39 & 9.15 & +0.001 & 48.7 & 8.77 & 16.9 & 8.70 \\
\hline $\mathrm{Na} I$ & 5682.63 & 2.10 & -0.700 & 98.0 & 6.27 & 94.6 & 5.74 \\
\hline $\mathrm{Na} I$ & 6154.23 & 2.10 & -1.560 & 36.6 & 6.27 & 35.2 & 5.89 \\
\hline $\mathrm{Na} \mathrm{I}$ & 6160.75 & 2.10 & -1.260 & 57.0 & 6.29 & 55.4 & 5.87 \\
\hline Mg I & 4730.03 & 4.35 & -2.523 & 72.8 & 7.89 & 76.7 & 7.52 \\
\hline $\mathrm{Mg}$ I & 5711.09 & 4.35 & -1.833 & 105.3 & 7.61 & 126.5 & 7.32 \\
\hline $\mathrm{Mg}$ I & 6318.72 & 5.11 & -1.945 & 45.5 & 7.57 & 44.6 & 7.29 \\
\hline $\mathrm{Mg}_{\mathrm{I}}$ & 6319.24 & 5.11 & -2.165 & 30.8 & 7.55 & 30.1 & 7.30 \\
\hline $\mathrm{Mg}$ I & 6965.41 & 5.75 & -1.510 & 24.3 & 7.29 & 24.3 & 7.14 \\
\hline $\mathrm{Al} \mathrm{I}$ & 6696.02 & 3.14 & -1.347 & 37.3 & 6.25 & 46.3 & 6.05 \\
\hline Al I & 6698.67 & 3.14 & -1.647 & 21.0 & 6.22 & 27.2 & 6.04 \\
\hline Si I & 5690.43 & 4.93 & -1.769 & 53.4 & 7.53 & 34.7 & 7.13 \\
\hline Si I & 5701.10 & 4.93 & -1.581 & 38.5 & 7.10 & 26.7 & 6.79 \\
\hline Si I & 5772.15 & 5.08 & -1.358 & 52.3 & 7.23 & 33.9 & 6.86 \\
\hline Si I & 6125.02 & 5.61 & -1.464 & 31.3 & 7.46 & 17.1 & 7.12 \\
\hline Si I & 6142.48 & 5.62 & -1.295 & 34.1 & 7.34 & 19.5 & 7.02 \\
\hline $\mathrm{Si} \mathrm{I}$ & 6145.02 & 5.62 & -1.310 & 38.1 & 7.42 & 21.3 & 7.07 \\
\hline $\mathrm{Si}$ I & 6243.82 & 5.62 & -1.242 & 46.4 & 7.49 & 27.2 & 7.14 \\
\hline Si I & 6244.47 & 5.62 & -1.093 & 45.2 & 7.32 & 26.0 & 6.96 \\
\hline $\mathrm{Si}$ I & 6414.98 & 5.87 & -1.035 & 47.2 & 7.49 & 26.0 & 7.14 \\
\hline $\mathrm{Si} \mathrm{I}$ & 6741.63 & 5.98 & -1.428 & 16.0 & 7.37 & 04.1 & 6.79 \\
\hline Si I & 6848.58 & 5.86 & -1.524 & 17.0 & 7.39 & 07.2 & 7.01 \\
\hline Si I & 7405.77 & 5.61 & -0.313 & 90.2 & 7.11 & 65.5 & 6.74 \\
\hline $\mathrm{K}_{\mathrm{I}}$ & 7698.98 & 0.00 & -0.170 & 155.3 & 5.27 & 185.3 & 4.73 \\
\hline $\mathrm{Ca} \mathrm{I}$ & 5867.56 & 2.93 & -1.570 & 25.5 & 6.33 & 29.6 & 6.02 \\
\hline $\mathrm{Ca} \mathrm{I}$ & 6161.30 & 2.52 & -1.266 & 63.3 & 6.30 & 77.7 & 5.97 \\
\hline $\mathrm{Ca} \mathrm{I}$ & 6166.44 & 2.52 & -1.142 & 69.0 & 6.26 & 83.7 & 5.91 \\
\hline $\mathrm{Ca} \mathrm{I}$ & 6169.04 & 2.52 & -0.797 & 90.9 & 6.26 & 108.1 & 5.85 \\
\hline $\mathrm{Ca} \mathrm{I}$ & 6169.56 & 2.53 & -0.478 & 110.0 & 6.21 & 132.5 & 5.78 \\
\hline $\mathrm{Ca} \mathrm{I}$ & 6455.60 & 2.52 & -1.340 & 55.7 & 6.24 & 67.6 & 5.90 \\
\hline $\mathrm{Ca} \mathrm{I}$ & 6493.78 & 2.52 & -0.109 & 123.8 & 5.98 & 148.6 & 5.53 \\
\hline $\mathrm{Ca} \mathrm{I}$ & 6499.65 & 2.52 & -0.818 & 85.4 & 6.18 & 96.4 & 5.73 \\
\hline Sc II & 6245.64 & 1.51 & -1.030 & 34.3 & 3.06 & 23.9 & 2.73 \\
\hline Sc II & 6604.60 & 1.36 & -1.309 & 35.1 & 3.18 & 24.1 & 2.83 \\
\hline Ti I & 5022.87 & 0.83 & -0.434 & 72.5 & 4.79 & 102.4 & 4.44 \\
\hline Ti I & 5024.84 & 0.82 & -0.602 & 68.9 & 4.88 & 94.4 & 4.48 \\
\hline Ti I & 5866.45 & 1.07 & -0.840 & 46.8 & 4.89 & 76.8 & 4.62 \\
\hline Ti I & 6091.17 & 2.27 & -0.423 & 14.5 & 4.93 & 30.4 & 4.78 \\
\hline Ti I & 6098.66 & 3.06 & -0.010 & 05.4 & 4.81 & 10.5 & 4.68 \\
\hline Ti I & 6258.10 & 1.44 & -0.355 & 51.6 & 4.84 & 77.3 & 4.53 \\
\hline Ti I & 6261.10 & 1.43 & -0.479 & 48.8 & 4.90 & 74.5 & 4.60 \\
\hline Ti II & 5154.07 & 1.57 & -1.750 & 72.2 & 5.01 & 77.7 & 4.90 \\
\hline Ti II & 5336.79 & 1.58 & -1.590 & 70.6 & 4.82 & 59.9 & 4.45 \\
\hline Ti II & 5381.02 & 1.57 & -1.920 & 57.3 & 4.87 & 46.8 & 4.55 \\
\hline V I & 5737.06 & 1.06 & -0.740 & 12.0 & 3.98 & 28.5 & 3.75 \\
\hline V I & 6081.44 & 1.05 & -0.579 & 13.8 & 3.85 & 33.9 & 3.66 \\
\hline V I & 6090.21 & 1.08 & -0.062 & 32.7 & 3.85 & 58.3 & 3.57 \\
\hline V I & 6111.65 & 1.04 & -0.715 & 11.5 & 3.89 & 31.5 & 3.74 \\
\hline V I & 6224.53 & 0.29 & -2.010 & 05.6 & 4.08 & 21.7 & 3.97 \\
\hline V I & 6243.11 & 0.30 & -0.980 & 29.3 & 3.90 & 71.0 & 3.78 \\
\hline
\end{tabular}


Table A.1. continued

\begin{tabular}{|c|c|c|c|c|c|c|c|}
\hline Ion & $\begin{array}{l}\lambda \\
(\AA)\end{array}$ & $\begin{array}{c}\chi \\
(\mathrm{eV}) \\
\end{array}$ & $\begin{array}{c}\log g f \\
(-)\end{array}$ & $\begin{array}{l}\text { The Sun } \\
\mathrm{EW}_{\odot}\end{array}$ & $\log N_{\odot}$ & $\begin{array}{l}\text { Kepler-444 } \\
\text { EW }\end{array}$ & $\log N$ \\
\hline V I & 6251.83 & 0.29 & -1.340 & 15.4 & 3.89 & 45.9 & 3.75 \\
\hline Cr I & 5702.31 & 3.45 & -0.667 & 25.4 & 5.83 & 23.2 & 5.37 \\
\hline Cr I & 5783.06 & 3.32 & -0.500 & 32.0 & 5.68 & 30.8 & 5.22 \\
\hline Cr I & 5783.85 & 3.32 & -0.295 & 42.3 & 5.67 & 30.8 & 5.20 \\
\hline Cr I & 5787.92 & 3.32 & -0.083 & 45.4 & 5.51 & 44.4 & 5.03 \\
\hline Cr I & 6330.09 & 0.94 & -2.920 & 26.3 & 5.61 & 40.3 & 5.18 \\
\hline Cr I & 7400.25 & 2.90 & -0.111 & 74.2 & 5.54 & 86.1 & 5.11 \\
\hline $\mathrm{Fe} \mathrm{I}$ & 4779.44 & 3.42 & -2.000 & 40.5 & 7.25 & 39.0 & 6.77 \\
\hline Fe I & 4788.76 & 3.24 & -1.800 & 65.5 & 7.29 & 62.2 & 6.69 \\
\hline $\mathrm{Fe} \mathrm{I}$ & 5054.64 & 3.64 & -1.900 & 39.8 & 7.33 & 38.3 & 6.88 \\
\hline $\mathrm{Fe} \mathrm{I}$ & 5322.04 & 2.28 & -2.800 & 59.8 & 7.24 & 58.8 & 6.61 \\
\hline $\mathrm{Fe} \mathrm{I}$ & 5379.57 & 3.69 & -1.500 & 60.6 & 7.33 & 53.6 & 6.75 \\
\hline $\mathrm{Fe} I$ & 5522.45 & 4.21 & -1.600 & 42.3 & 7.52 & 33.5 & 7.00 \\
\hline $\mathrm{Fe} \mathrm{I}$ & 5543.94 & 4.22 & -1.100 & 61.5 & 7.46 & 52.7 & 6.91 \\
\hline $\mathrm{Fe} I$ & 5546.50 & 4.37 & -1.300 & 50.6 & 7.58 & 41.3 & 7.06 \\
\hline $\mathrm{Fe} \mathrm{I}$ & 5546.99 & 4.22 & -1.900 & 27.1 & 7.58 & 24.8 & 7.20 \\
\hline $\mathrm{Fe} I$ & 5560.21 & 4.43 & -1.200 & 51.3 & 7.53 & 39.7 & 6.98 \\
\hline Fe I & 5587.57 & 4.14 & -1.900 & 38.9 & 7.69 & 32.1 & 7.20 \\
\hline $\mathrm{Fe} \mathrm{I}$ & 5646.68 & 4.26 & -2.500 & 7.4 & 7.53 & 05.0 & 7.04 \\
\hline $\mathrm{Fe} I$ & 5651.47 & 4.47 & -2.000 & 18.9 & 7.70 & 12.7 & 7.20 \\
\hline $\mathrm{Fe} \mathrm{I}$ & 5652.32 & 4.26 & -1.900 & 26.1 & 7.64 & 18.3 & 7.11 \\
\hline $\mathrm{Fe} I$ & 5661.35 & 4.28 & -1.700 & 22.3 & 7.35 & 15.4 & 6.84 \\
\hline $\mathrm{Fe} \mathrm{I}$ & 5667.52 & 4.18 & -1.600 & 50.4 & 7.66 & 36.2 & 7.04 \\
\hline $\mathrm{Fe} \mathrm{I}$ & 5679.02 & 4.65 & -0.900 & 58.6 & 7.58 & 46.9 & 7.04 \\
\hline $\mathrm{Fe} \mathrm{I}$ & 5680.24 & 4.19 & -2.600 & 11.0 & 7.73 & 07.8 & 7.25 \\
\hline $\mathrm{Fe} I$ & 5731.76 & 4.26 & -1.300 & 56.9 & 7.57 & 46.0 & 7.01 \\
\hline $\mathrm{Fe} I$ & 5741.85 & 4.26 & -1.900 & 31.2 & 7.64 & 23.4 & 7.14 \\
\hline $\mathrm{Fe} \mathrm{I}$ & 5752.03 & 4.55 & -1.200 & 53.8 & 7.66 & 42.7 & 7.13 \\
\hline $\mathrm{Fe} I$ & 5775.08 & 4.22 & -1.300 & 57.9 & 7.55 & 47.3 & 6.98 \\
\hline $\mathrm{Fe} \mathrm{I}$ & 5778.45 & 2.59 & -3.500 & 22.0 & 7.44 & 21.2 & 6.92 \\
\hline $\mathrm{Fe} I$ & 5809.22 & 3.88 & -1.800 & 48.3 & 7.59 & 39.1 & 7.03 \\
\hline $\mathrm{Fe} \mathrm{I}$ & 6079.00 & 4.65 & -1.100 & 45.9 & 7.55 & 32.4 & 7.00 \\
\hline $\mathrm{Fe} \mathrm{I}$ & 6085.26 & 2.76 & -3.100 & 42.5 & 7.64 & 59.1 & 7.39 \\
\hline $\mathrm{Fe} \mathrm{I}$ & 6098.24 & 4.56 & -1.900 & 16.5 & 7.57 & 10.0 & 7.05 \\
\hline $\mathrm{Fe} \mathrm{I}$ & 6151.62 & 2.18 & -3.300 & 48.3 & 7.37 & 49.6 & 6.80 \\
\hline $\mathrm{Fe} I$ & 6159.37 & 4.61 & -2.000 & 12.2 & 7.56 & 07.3 & 7.04 \\
\hline $\mathrm{Fe} \mathrm{I}$ & 6165.36 & 4.14 & -1.500 & 43.7 & 7.37 & 33.8 & 6.83 \\
\hline $\mathrm{Fe} I$ & 6187.99 & 3.94 & -1.700 & 46.1 & 7.47 & 36.6 & 6.92 \\
\hline $\mathrm{Fe} \mathrm{I}$ & 6220.78 & 3.88 & -2.500 & 19.1 & 7.58 & 14.0 & 7.06 \\
\hline $\mathrm{Fe} I$ & 6226.73 & 3.88 & -2.200 & 28.2 & 7.56 & 20.5 & 7.02 \\
\hline $\mathrm{Fe} \mathrm{I}$ & 6229.23 & 2.85 & -2.800 & 35.7 & 7.30 & 32.7 & 6.76 \\
\hline $\mathrm{Fe} \mathrm{I}$ & 6240.65 & 2.22 & -3.200 & 47.3 & 7.31 & 47.6 & 6.74 \\
\hline $\mathrm{Fe} \mathrm{I}$ & 6293.92 & 4.84 & -1.700 & 13.2 & 7.56 & 07.4 & 7.04 \\
\hline $\mathrm{Fe} \mathrm{I}$ & 6380.74 & 4.19 & -1.400 & 51.2 & 7.46 & 40.3 & 6.90 \\
\hline $\mathrm{Fe} I$ & 6392.54 & 2.28 & -4.000 & 17.7 & 7.53 & 19.3 & 7.04 \\
\hline $\mathrm{Fe} \mathrm{I}$ & 6597.56 & 4.79 & -1.100 & 43.9 & 7.57 & 31.6 & 7.06 \\
\hline $\mathrm{Fe} I$ & 6608.02 & 2.28 & -4.000 & 17.0 & 7.49 & 19.6 & 7.04 \\
\hline $\mathrm{Fe} \mathrm{I}$ & 6627.54 & 4.55 & -1.700 & 27.1 & 7.62 & 19.3 & 7.14 \\
\hline $\mathrm{Fe} I$ & 6703.57 & 2.76 & -3.200 & 35.9 & 7.54 & 34.3 & 7.02 \\
\hline $\mathrm{Fe} \mathrm{I}$ & 6710.32 & 1.49 & -4.900 & 15.1 & 7.49 & 18.1 & 6.96 \\
\hline $\mathrm{Fe} \mathrm{I}$ & 6713.74 & 4.80 & -1.600 & 20.7 & 7.62 & 13.0 & 7.13 \\
\hline $\mathrm{Fe} \mathrm{I}$ & 6716.22 & 4.58 & -1.900 & 15.5 & 7.58 & 06.2 & 6.87 \\
\hline $\mathrm{Fe} \mathrm{I}$ & 6725.35 & 4.10 & -2.300 & 17.9 & 7.57 & 11.5 & 7.02 \\
\hline $\mathrm{Fe} I$ & 6726.67 & 4.61 & -1.100 & 25.8 & 7.10 & 32.9 & 6.95 \\
\hline $\mathrm{Fe} \mathrm{I}$ & 6733.15 & 4.64 & -1.600 & 25.9 & 7.58 & 16.2 & 7.04 \\
\hline Fe I & 6739.52 & 1.56 & -4.800 & 11.3 & 7.32 & 14.4 & 6.83 \\
\hline $\mathrm{Fe} I$ & 6752.72 & 4.64 & -1.300 & 35.1 & 7.49 & 23.6 & 6.96 \\
\hline Fe II & 4620.52 & 2.83 & -3.300 & 51.3 & 7.39 & 23.9 & 6.87 \\
\hline
\end{tabular}


C. E. Mack III et al: PEPSI deep spectra. III.

Table A.1. continued

\begin{tabular}{|c|c|c|c|c|c|c|c|}
\hline Ion & $\begin{array}{c}\lambda \\
(\AA)\end{array}$ & $\begin{array}{c}\chi \\
(\mathrm{eV})\end{array}$ & $\begin{array}{c}\log g f \\
(-)\end{array}$ & $\begin{array}{l}\text { The Sun } \\
\mathrm{EW}_{\odot}\end{array}$ & $\log N_{\odot}$ & $\begin{array}{l}\text { Kepler-444 } \\
\text { EW }\end{array}$ & $\log N$ \\
\hline Fe II & 5197.58 & 3.23 & -2.300 & 79.0 & 7.36 & 44.5 & 6.77 \\
\hline $\mathrm{Fe}$ II & 5234.62 & 3.22 & -2.300 & 83.3 & 7.37 & 47.0 & 6.74 \\
\hline Fe II & 5264.81 & 3.23 & -3.100 & 47.1 & 7.47 & 20.3 & 7.00 \\
\hline $\mathrm{Fe}$ II & 5414.07 & 3.22 & -3.600 & 28.7 & 7.56 & 09.4 & 7.09 \\
\hline Fe II & 5425.26 & 3.20 & -3.400 & 41.0 & 7.56 & 19.3 & 7.19 \\
\hline $\mathrm{Fe}$ II & 6149.26 & 3.89 & -2.800 & 36.2 & 7.55 & 10.6 & 7.02 \\
\hline Fe II & 6247.56 & 3.89 & -2.400 & 51.7 & 7.47 & 19.0 & 6.94 \\
\hline $\mathrm{Fe}$ II & 7711.72 & 3.90 & -2.700 & 45.3 & 7.54 & 13.8 & 7.00 \\
\hline Co I & 5352.04 & 3.58 & +0.060 & 25.1 & 4.57 & 22.5 & 4.19 \\
\hline Co I & 5301.04 & 1.71 & -2.000 & 20.8 & 4.97 & 30.9 & 4.66 \\
\hline Co I & 5647.23 & 2.28 & -1.560 & 14.4 & 4.88 & 19.5 & 4.56 \\
\hline Co I & 6093.14 & 1.74 & -2.440 & 09.6 & 4.99 & 14.6 & 4.68 \\
\hline Co I & 6814.94 & 1.96 & -1.900 & 19.3 & 4.98 & 27.4 & 4.67 \\
\hline $\mathrm{Ni} \mathrm{I}$ & 5748.35 & 1.68 & -3.260 & 28.5 & 6.19 & 28.6 & 5.71 \\
\hline $\mathrm{Ni} I$ & 5754.66 & 1.94 & -2.330 & 76.6 & 6.44 & 74.5 & 5.83 \\
\hline Ni I & 5760.83 & 4.11 & -0.800 & 35.1 & 6.24 & 23.4 & 5.75 \\
\hline $\mathrm{Ni} \mathrm{I}$ & 5846.99 & 1.68 & -3.210 & 22.7 & 6.00 & 22.2 & 5.51 \\
\hline $\mathrm{Ni}$ I & 6108.11 & 1.68 & -2.450 & 64.6 & 6.04 & 63.6 & 5.47 \\
\hline $\mathrm{Ni} \mathrm{I}$ & 6111.07 & 4.09 & -0.870 & 34.2 & 6.26 & 22.5 & 5.77 \\
\hline $\mathrm{Ni}$ I & 6128.96 & 1.68 & -3.330 & 24.8 & 6.15 & 24.4 & 5.67 \\
\hline Ni I & 6130.13 & 4.27 & -0.960 & 21.8 & 6.24 & 12.6 & 5.74 \\
\hline $\mathrm{Ni}$ I & 6133.96 & 4.09 & -1.830 & 05.8 & 6.27 & 04.3 & 5.92 \\
\hline $\mathrm{Ni} \mathrm{I}$ & 6175.36 & 4.09 & -0.559 & 48.2 & 6.21 & 34.4 & 5.70 \\
\hline $\mathrm{Ni} \mathrm{I}$ & 6176.81 & 4.09 & -0.260 & 62.9 & 6.16 & 47.3 & 5.61 \\
\hline $\mathrm{Ni} \mathrm{I}$ & 6177.24 & 1.83 & -3.500 & 14.8 & 6.19 & 13.6 & 5.70 \\
\hline $\mathrm{Ni} \mathrm{I}$ & 6186.71 & 4.11 & -0.960 & 30.8 & 6.29 & 19.1 & 5.78 \\
\hline $\mathrm{Ni}$ I & 6204.60 & 4.09 & -1.100 & 21.2 & 6.19 & 13.1 & 5.71 \\
\hline $\mathrm{Ni} \mathrm{I}$ & 6223.98 & 4.11 & -0.910 & 27.5 & 6.17 & 17.3 & 5.68 \\
\hline $\mathrm{Ni}$ I & 6230.09 & 4.11 & -1.260 & 21.0 & 6.36 & 13.2 & 5.90 \\
\hline $\mathrm{Ni} \mathrm{I}$ & 6327.59 & 1.68 & -3.150 & 38.2 & 6.25 & 38.6 & 5.76 \\
\hline $\mathrm{Ni}$ I & 6370.34 & 3.54 & -1.940 & 12.8 & 6.23 & 09.1 & 5.80 \\
\hline $\mathrm{Ni}$ I & 6378.25 & 4.15 & -0.830 & 31.9 & 6.22 & 20.7 & 5.74 \\
\hline $\mathrm{Ni} \mathrm{I}$ & 6635.12 & 4.42 & -0.820 & 25.4 & 6.31 & 15.8 & 5.86 \\
\hline Ni I & 6643.63 & 1.68 & -2.300 & 93.9 & 6.38 & 93.9 & 5.74 \\
\hline $\mathrm{Ni} \mathrm{I}$ & 6767.77 & 1.83 & -2.170 & 78.4 & 6.11 & 76.2 & 5.50 \\
\hline $\mathrm{Ni}$ I & 6842.04 & 3.66 & -1.480 & 26.4 & 6.26 & 18.3 & 5.79 \\
\hline Mn I & 5432.55 & 0.00 & -4.382 & 52.2 & 5.34 & 79.6 & 4.83 \\
\hline Mn I & 6013.51 & 3.07 & -0.765 & 87.2 & 5.36 & 81.3 & 4.76 \\
\hline Mn I & 6021.82 & 3.08 & -0.537 & 95.1 & 5.63 & 88.5 & 4.90 \\
\hline $\mathrm{Zn} \mathrm{I}$ & 4722.16 & 4.03 & -0.338 & 67.9 & 4.43 & 52.2 & 4.02 \\
\hline $\mathrm{Zn}$ I & 4810.53 & 4.08 & -0.137 & 71.9 & 4.35 & 56.9 & 3.94 \\
\hline Y II & 5200.40 & 0.99 & -0.570 & 38.0 & 2.11 & 22.6 & 1.57 \\
\hline
\end{tabular}

